Conditioned Withdrawal Responses with Shock as the Conditioning Stimulus in Adult Human Subjects. (Psychol. Bulletin, vol. xxxi, p. I I , Feb., 1934.) Razran, G. H. S.

The withdrawal response to an electric shock is of great interest in some special problems, but its general value as a method for the discovery of the laws of conditioning in adult human beings is very limited. Many of the recorded observations indicate that the experiments have not normally yielded results of pure conditionability. While conditioned responses are not brought about by consciousness, attention and the like, they are affected, much more than the so-called native responses, by subjective processes, and their true course is obscured by the latter. The supposition that these subjective processes are themselves governed by laws which are the same, identical, similar, or derivable from the laws of conditioning does not remove the difficulty. M. Hamblin Smith.

The Irradiation of a Tactile Conditioned Reflex in Man. (Journ. Comp. Psychol., vol. xvii, p. 47, Feb., 1934.) Bass, M. J., and Hull, C. L.

The points of stimulation in these experiments were four in number-the left shoulder, the left side of the small of the back, the middle of the left thigh and the middle of the left calf. There is a definite spread of cutaneous conditioned excitatory tendency from the shoulder towards the feet, and from the calf towards the head. There is also a definite analogous spread of the inhibition resulting from experimental extinction. In both cases the spread takes the form of a gradient, the magnitude of the tendency diminishing progressively with distance from the point of origin. The results are in accord with the findings of Anrep, in spite of wide differences in the technique of the experiments. The authors express scepticism concerning the validity of Pavlov's neurological speculations as distinguished from his experimental findings. M. Hamblin Smith.

The Physiology of Sleep. X: The Effect of Alcohol and Caffeine on Motility and Body Temperature During Sleep. (Amer. Journ. Physiol., vol. cvi, p. 478, 1933.) Mullin, F.J., Kleitman, N., and Cooperman, N.R.

In human male subjects $300-375$ c.c. of $19 \%$ alcohol taken one hour before retiring causes a lowering of motility and body temperature during the first half of the night, with an increase in both during the last part of the night's sleep. Large doses of caffeine (4-6 gr.) produce a marked increase in motility and body temperature during sleep. J. F. LymaN (Chem. Abstr.).

Behaviour in Its Relation to the Development of the Brain. Part I. (Bull. Neur. Inst. N.Y., vol. i, p. 229, June, 1931.) Tilney, F. R., and Kubie, L. S.

The authors aim to demonstrate the correlation of behaviour and the development of the brain. Their chief purpose is to establish an explanatory basis for adjustive reactions in terms of cerebral structure. The plan of the investigation is outlined, and is based on the fact that all structures of the body attain adequate differentiation before they are capable of specialized reactions. The maturing process in six different species-opossum, rat, guinea-pig, pig, cat and man - are to be followed in two concurrent studies: (1) The development of the brain ; (2) the development of behaviour, with the object of establishing chronological relations between the developmental processes.

In the structural investigation three methods are employed : (I) Organogenetic studies by means of the Born method of reconstruction ; (2) histogenetic studies ; and (3) myelinogenetic studies. The results of the structural investigation up to June, I931, are reported, including a review of the developmental processes in the end-brain of the domestic cat, which result in the formation of the paleocortex, archicortex and neocortex. It shows that these processes pass successively through a three-layer and a four-layer stage, recapitulating respectively icthyopsid and reptilian conditions before the ultimate mammalian six-layer cortex is attained. 
It also traces the development of the tract-beds, which have neocortical connections. It concludes with a formula upon which the functional evolution of the neocortex in this mammalian species (cat) appears to be based, and suggests that the same formula - with certain modifications-may be applied to all mammals. The formula is that the higher evolutional levels in the cortex depend on $(a)$ the projection of the senses upon special neocortical areas, in which process general body-sense precedes the special senses ; $(b)$ the bilateral association of these senses in the two hemispheres, in which again general body-sense takes precedence over the special senses ; $(c)$ the intimate associations and elaborations within each type of sense as well as inter-associations between all of the senses; $(d)$ the efferent projection of sensory associations into the somatic effector activities of the animal's behaviour.

Part II : Correlation Between the Development of the Brain and Behaviour in the Albino Rat from Embryonic States to Maturity. (Bull. Neur. Inst. N.Y., vol. iii, p. 252, June, 1933.) Tilney, F. R.

This paper is based on data obtained by serial sections of the central nervous system of albino rats from the $8-\mathrm{mm}$. embryo to the adult, and on observations upon the fœtal and post-natal behaviour of the same animal. In the pre-natal stages the fœtuses were floated out of the uterus into warm saline solution, and the reaction studied with the umbilical cord still attached to the placenta in sit $\hat{u}$. Somatic reactions only were studied, the investigation of splanchnic activities being postponed for subsequent consideration. The analysis of reactions proceeded upon the assumption that a structural adequacy in the nervous system is essential to the several activities which make their appearance in the successive stages of development, and that such adequacy is founded upon the capacity of the neural apparatus for afferent conduction, central integration and efferent transmission. Three significant phases in the development of the cortex were distinguished, and each phase represents a distinct period in the up-building of behaviour, and serves as an index of the relative degree of specialization in the entire nervous system.

(I) The first phase (general cortical differentiation) covers the embryonic and fotal periods. The animal's reactions are at first limited to circumscribed muscular twitchings, then more definitely reflex qualities appear, and finally, simple but distinctly integrated responses to stimulation occur. In this phase, a series of migratory laminations from the mantle layer, with cellular differentiation in the strata thus formed, results in the formation of a six-layered neocortex about the time of birth. Cortical differentiation occurs in the olfactory bulb and in the palæocortical and archicortical portions of the end-brain. Cellular specialization and maturation is much more marked in the spinal cord and brain-stem than elsewhere, and in this pre-natal period, structural immaturity in the hemisphere, interbrain, midbrain and cerebellum is sufficient to warrant the exclusion of these structures from any part in the control of behaviour. Such control must be, at this stage, attributed to the spinal cord and caudal portion of the brain-stem.

(2) The second phase (divisional cortical differentiation) begins at birth and continues into the fifth or sixth post-natal day. Such activities as breathing, crawling, righting and sucking manifest themselves during this time, and such reactions are attributed to the spinal cord, brain-stem and tegmentum, as the structure of higher centres is as yet too immature to justify allocation of functional activity to them. Late in this phase the, young rat develops greater vigour in its postural and nutritional activities, and ineffectual attempts at scratching are made. Reasons for associating these later reactions with developments in interbrain, corpus striatum and olfactory cortex are discussed.

(3) The third phase (local cortical differentiation) begins on or about the fifth post-natal day, and is well advanced by the tenth day. It results in the differentiation of thirty-one distinct cortical areas. During this phase the subcortical nuclei, the interbrain and the cerebellum attain their full development much earlier 
than the neocortex. Myelinization slowly advances in nerve-roots, spinal cord, caudal portions of the brain-stem and cerebellum. Later it affects the midbrain and finally the cerebral hemisphere. While local cortical differentiation is in process, the animal increases the number and effectiveness of its reactions until it displays all the behaviour characteristics of its kind. In many respects the cortical differentiation in the adult rat is extremely primitive, and this accounts for the comparatively limited rôle of the cortex in the control of the somatic behaviour of the albino rat.

J. L. FAULL.

\section{Psychology and Psychopathology.}

Trends in Modern Psychology. (Psychol. Bull., vol. xxxi, p. 81, Feb., 1934.) Goodenough, F. L.

This paper is based upon the proportionate number of publications listed in the Psychological Index and noted in Psychological Abstracts. The curve for educational psychology rises steadily. There has been a marked increase in papers on industrial and personal problems. The increase in papers upon childhood and adolescence has been very marked, and this also applies to mental tests; the increase in the latter subject has been greater in America than in other countries. Questions of statistical method have increased during recent years. Publications on sensation and perception have not kept pace with the increase in other topics. Papers on the nervous system show an absolute decrease. The employment of philosophical terms in the titles of papers tends to diminish in frequency.

M. Hamblin Smith.

A Quantitative Study of Eidetic Imagery. (Brit. Journ. Educ. Psychol., vol. iv, p. 56, Feb., 1934.) Teasdale, H.

The author found that over $60 \%$ of children between ro-14 years of age, in the locality investigated, failed to get any image on a grey background, after regarding a silhouette picture with non-fixation. Less than $10 \%$ of the children tested had images rich in detail and of several minutes' duration. Among those who did get images there was a gradation from very weak to very strong images. When only the richest and most stable images were counted as eidetic images, it was found that eidetic ability was most frequent at the youngest ages tested. If the standard was made a little lower, so that images of a rather inferior type were included, then eidetic images were found to be most frequent from II-I 2 years. It appears that there are two types of eidetic imagery, one which is very prevalent among young children and decreases with increasing age, and one which is not so rich in detail, which does not show such marked divergencies from normal after-images, and which becomes more frequent with increasing age up to 14 years. G. W. T. H. Fleming.

Noises and Colours in Relation to Mental Hygiene [Los ruidos y los colores desde el punto de vista de la higiene mental]. (La Semana Méd., vol. xli, p. 252, Jan. 18, 1934.) Gorriti, F.

The plague of noise, so marked in great cities, is highly prejudicial in certain cases, and should be made the subject of mental hygiene propaganda. Constant noises may precipitate the occurrence of psychoses and may produce or aggravate nervous states. Delusions of persecution may be traced to the influence of oftrepeated noises. The influence of colours upon mental state is well known, and has long been employed in theatres. We should make use of this influence in mental hospitals. Wards for the treatment of maniacal patients should have windows of blue glass, while red glass should be used for melancholics and dements. By planting gardens with flowers of these respective colours, we may extend the therapeutic use of colour to patients when out of doors.

M. Hamblin Smith. 